\title{
Subclass of $p$-valent Function with Negative Coefficients Applying Generalized Al-Oboudi Differential Operator
}

\author{
Timilehin G. Shaba ${ }^{1, *}$, Abd'gafar T. Tiamiyu ${ }^{2}$, \\ Ismaila O. Ibrahim ${ }^{3}$ and Abdullahi A. Ibrahim ${ }^{4}$ \\ ${ }^{1}$ Department of Mathematics, University of Ilorin, P. M. B. 1515, Ilorin, Nigeria \\ e-mail: shabatimilehin@gmail.com \\ ${ }^{2}$ Department of Mathematics, Federal University of Technology, Minna, Nigeria \\ e-mail: abdgafartunde@yahoo.com \\ ${ }^{3}$ Department of Mathematical science, University of Maiduguri, Nigeria \\ e-mail: ibrahimismailaomeiza@gmail.com \\ ${ }^{4}$ Department of Mathematics, Baze University, Nigeria \\ e-mail: abdullahi.ibrahim@bazeuniversity.edu.ng
}

\begin{abstract}
In this paper we introduce a new subclass $\mathcal{R}^{*}(p, g, \psi, \varrho, \beta, \phi, \gamma, \zeta)$ of $p$-valent functions with negative coefficient defined by Hadamard product associated with a generalized differential operator. Radii of close-to-convexity, starlikeness and convexity of the class $\mathcal{R}^{*}(p, g, \psi, \varrho, \beta, \phi, \gamma, \zeta)$ are obtained. Also, distortion theorem, growth theorem and coefficient inequalities are established.
\end{abstract}

\section{Introduction and Definitions}

Let $\mathcal{G}$ be class of functions $f(z)$ of the form

$$
f(z)=z+\sum_{w=2}^{\infty} l_{w} z^{w}
$$

Received: December 1, 2020; Accepted: January 2, 2021

2010 Mathematics Subject Classification: Primary 30C45; Secondary 30C50.

Keywords and phrases: close to convexity, univalent functions, Hadamard product, convexity, Al-Oboudi differential operator, starlike function.

${ }^{*}$ Corresponding author 
which are holomorphic in the open unit disk $\triangle=\{z \in \mathbb{C}:|z|<1\}$.

For $f(z)$ belongs to $\mathcal{G}$, Opoola [6] (see also [11, 12]) has introduced the following differential operator:

$$
\begin{gathered}
D_{\phi, \zeta}^{0, \gamma} f(z)=f(z) \\
D_{\phi, \zeta}^{1, \gamma} f(z)=(1+(\phi-\gamma-1) \zeta) f(z)-z(\gamma-\phi) \zeta+z \zeta f^{\prime}(z)=D_{\phi, \zeta}^{\gamma} f(z), \\
D_{\phi, \zeta}^{2, \gamma} f(z)=D_{\phi, \zeta}^{\gamma}\left(D_{\phi, \zeta}^{1, \gamma} f(z)\right), \\
D_{\phi, \zeta}^{h, \gamma} f(z)=D_{\phi, \zeta}^{\gamma}\left(D_{\phi, \zeta}^{h+1, \gamma} f(z)\right),
\end{gathered}
$$

if $f(z)$ is given by (1.1), then by $(1.2)$ and $(1.3)$, we see that

$$
D_{\phi, \zeta}^{h, \gamma} f(z)=z+\sum_{w=2}^{\infty}(1+(w+\phi-\gamma-1) \zeta)^{h} l_{w} z^{w},
$$

where $0 \leq \phi \leq \gamma, \zeta \geq 0$ and $h \in \mathbb{N}_{0}=\{0,1,2,3, \cdots\}$.

Let $\mathcal{T}$ denote the subclass of $\mathcal{G}$ consisting of the form

$$
f(z)=z-\sum_{w=2}^{\infty} l_{w} z^{w},
$$

where $l_{w} \geq 0$ and $w \in \mathbb{N}$. This class has introduced and studied by Silverman [9].

The Hadamard product of two power series

$$
f(z)=z-\sum_{w=2}^{\infty} l_{w} z^{w}, \quad g(z)=z-\sum_{w=2}^{\infty} j_{w} z^{w}
$$

and it is defined in $\mathcal{T}$ as follows:

$$
(f * g)=f(z) * g(z)=f(z)=z-\sum_{w=2}^{\infty} l_{w} j_{w} z^{w} .
$$

Let $\mathcal{G}_{p}$ denote the class of functions of the form

$$
f(z)=z^{p}+\sum_{w=1}^{\infty} l_{p+w} z^{p+w}
$$


that are holomorphic and $p$-valent in $|z|<1$.

Also let $\mathcal{T}_{p}$ denote the subclass of $\mathcal{G}_{p}$ consisting of functions that can be expressed as

$$
f(z)=z^{p}-\sum_{w=1}^{\infty} l_{p+w} z^{p+w} .
$$

The Hadamard product of two power series

$$
f(z)=z^{p}-\sum_{w=1}^{\infty} l_{p+w} z^{p+w}, \quad g(z)=z^{p}-\sum_{w=1}^{\infty} j_{p+w} z^{p+w}
$$

and it is defined in $\mathcal{T}_{p}$ as follows:

$$
(f * g)=f(z) * g(z)=f(z)=z-\sum_{w=1}^{\infty} l_{p+w} j_{p+w} z^{p+w} .
$$

From the above differential operator, the convolution of two power series $f(z)$ and $g(z)$ is given by

$$
D_{\phi, \zeta, p}^{h, \gamma}(f * g)(z)=p^{h} z^{p}-\sum_{w=1}^{\infty}(1+(p+w+\phi-\gamma-1) \zeta)^{h} l_{p+w} j_{p+w} z^{p+w},
$$

where $p \in \mathbb{N}=\{1,2,3, \cdots\}$. Motivated by [2], [10], 7], we define a new subclass $\mathcal{R}^{*}(p, g, \psi, \varrho, \beta, \phi, \gamma, \zeta)$ of the class $\mathcal{T}_{p}$.

Definition 1.1. For $0 \leq \psi<1, \varrho \geq 0$ and $0 \leq \phi \leq \gamma, 0 \leq \beta \leq \frac{1}{2}, \zeta \geq 0$, we let $\mathcal{R}^{*}(p, g, \psi, \varrho, \beta, \phi, \gamma, \zeta)$ be subclass of the class $\mathcal{T}_{p}$ consisting of functions of the form (1.7) and satisfying the analytic criterion

$$
\begin{array}{r}
\Re\left\{\frac{z D_{\phi, \zeta, p}^{h, \gamma}(f * g)^{\prime}(z)}{D_{\phi, \zeta, p}^{h, \gamma}(f * g)(z)}+\beta \frac{z^{2} D_{\phi, \zeta, p}^{h, \gamma}(f * g)^{\prime \prime}(z)}{D_{\phi, \zeta, p}^{h, \gamma}(f * g)(z)}-\psi\right\} \geq \varrho \mid \frac{z D_{\phi, \zeta, p}^{h, \gamma}(f * g)^{\prime}(z)}{D_{\phi, \zeta, p}^{h, \gamma}(f * g)(z)} \\
+\beta \frac{z^{2} D_{\phi, \zeta, p}^{h, \gamma}(f * g)^{\prime \prime}(z)}{D_{\phi, \zeta, p}^{h, \gamma}(f * g)(z)}-1 \mid .
\end{array}
$$

The main purpose of this paper is to investigate some geometric properties of the class $\mathcal{R}^{*}(p, g, \psi, \varrho, \beta, \phi, \gamma, \zeta)$ such as the coefficient bounds, growth and radii 
of starlikeness, distortion properties, convexity and close to convexity for the class $\mathcal{R}^{*}(p, g, \psi, \varrho, \beta, \phi, \gamma, \zeta)$. [5], 9], [1], 4], 8], [3], study the univalent functions for different classes.

\section{Coefficient Inequalities}

In the following theorem we obtain necessary and sufficient condition for a function $f(z)$ to be in the class $\mathcal{R}^{*}(p, g, \psi, \varrho, \beta, \phi, \gamma, \zeta)$. We have the following lemma useful for this work.

Lemma 2.1. [8] Let $\psi \geq 0$ and $\nu$ be any complex number. Then $\Re(\nu) \geq \psi$ if and only if

$$
|\nu-(1+\psi)|<|\nu+(1-\psi)| .
$$

Lemma 2.2. 8] Let $\varrho \geq 0,0 \leq \psi$ and $\theta \in \mathbb{R}$. Then

$$
\Re(\nu)>\varrho|\nu-1|+\psi
$$

if and only if

$$
\Re\left(\nu\left(1+\varrho e^{i \theta}\right)-\varrho e^{i \theta}\right)>\psi,
$$

where $\nu$ is a complex number.

Theorem 2.3. Let $f(z) \in \mathcal{T}_{p}$ be given by (1.7). Then $f(z) \in$ $\mathcal{R}^{*}(p, g, \psi, \varrho, \beta, \phi, \gamma, \zeta)$ if and only if

$$
\begin{array}{r}
\sum_{w=1}^{\infty}\{(p+k)[1+(p+w-1) \beta](1+\psi)-(\varrho+\psi)\}[1+(p+w+\phi-\gamma-1) \zeta]^{h} l_{p+w} j_{p+w} \\
\leq p^{h}[p-\psi] .
\end{array}
$$

The result is sharp for the function

$$
f(z)=z-\frac{p^{h}(p-\psi)+p^{h}(p-1) \varrho+\beta p^{h+1}(p-1) z^{h}(1-\varrho)}{\{(p+k)[1+(p+w-1) \beta](1+\psi)-(\varrho+\psi)\}} .
$$


Proof. If $f(z) \in \mathcal{R}^{*}(p, g, \psi, \varrho, \beta, \phi, \gamma, \zeta)$ and $|z|=1$, then by Definition 1.1

$$
\begin{aligned}
\Re\left\{\frac{z D_{\phi, \zeta, p}^{h, \gamma}(f * g)^{\prime}(z)}{D_{\phi, \zeta, p}^{h, \gamma}(f * g)(z)}+\beta \frac{z^{2} D_{\phi, \zeta, p}^{h, \gamma}(f * g)^{\prime \prime}(z)}{D_{\phi, \zeta, p}^{h, \gamma}(f * g)(z)}-\psi\right\} & \geq \varrho \mid \frac{z D_{\phi, \zeta, p}^{h, \gamma}(f * g)^{\prime}(z)}{D_{\phi, \zeta, p}^{h, \gamma}(f * g)(z)} \\
& +\beta \frac{z^{2} D_{\phi, \zeta, p}^{h, \gamma}(f * g)^{\prime \prime}(z)}{D_{\phi, \zeta, p}^{h, \gamma}(f * g)(z)}-1 \mid .
\end{aligned}
$$

Using Lemma 2.2, it is sufficient to show that

$$
\Re\left\{\left(\frac{z D_{\phi, \zeta, p}^{h, \gamma}(f * g)^{\prime}(z)}{D_{\phi, \zeta, p}^{h, \gamma}(f * g)(z)}+\beta \frac{z^{2} D_{\phi, \zeta, p}^{h, \gamma}(f * g)^{\prime \prime}(z)}{D_{\phi, \zeta, p}^{h, \gamma}(f * g)(z)}\right)\left(1+\varrho e^{i \theta}\right)-\varrho e^{i \theta}\right\} \geq \psi,
$$$$
\Re\left[\begin{array}{r}
{\left[z D_{\phi, \zeta, p}^{h, \gamma}(f * g)^{\prime}(z)\right]\left(1+\varrho e^{i \theta}\right)+\left[\beta z^{2} D_{\phi, \zeta, p}^{h, \gamma}(f * g)^{\prime \prime}(z)\right]\left(1+\varrho e^{i \theta}\right)} \\
-\varrho e^{i \theta} D_{\phi, \zeta, p}^{h, \gamma}(f * g)(z)
\end{array}\right]>\psi .
$$

For convenience, let

$$
\mathcal{A}(z)=\left[z D_{\phi, \zeta, p}^{h, \gamma}(f * g)^{\prime}(z)\right]\left(1+\varrho e^{i \theta}\right)+\left[\beta z^{2} D_{\phi, \zeta, p}^{h, \gamma}(f * g)^{\prime \prime}(z)\right]\left(1+\varrho e^{i \theta}\right)-\varrho e^{i \theta} D_{\phi, \zeta, p}^{h, \gamma}(f * g)(z)
$$

and

$$
\mathcal{B}(z)=D_{\phi, \zeta, p}^{h, \gamma}(f * g)(z)
$$

That is equation (2) is equivalent to

$$
\Re\left(\frac{\mathcal{A}(z)}{\mathcal{B}(z)}\right) \geq \psi
$$

applying Lemma 2.1

$$
\begin{aligned}
\left|\frac{\mathcal{A}(z)}{\mathcal{B}(z)}-(1+\psi)\right| \leq \mid \frac{\mathcal{A}(z)}{\mathcal{B}(z)} & +(1-\psi) \mid \\
& \Longrightarrow\left|\frac{\mathcal{A}(z)-(1+\psi) \mathcal{B}(z)}{\mathcal{B}(z)}\right|<\left|\frac{\mathcal{A}(z)+(1-\psi) \mathcal{B}(z)}{\mathcal{B}(z)}\right|
\end{aligned}
$$




$$
|\mathcal{A}(z)+(1-\psi) \mathcal{B}(z)|-|\mathcal{A}(z)-(1+\psi) \mathcal{B}(z)| .
$$

Now,

$$
\begin{aligned}
& |\mathcal{A}(z)+(1-\psi) \mathcal{B}(z)|=\mid\left[p^{h+1} z^{p}-\sum_{w=1}^{\infty}[1+(p+w+\phi-\gamma-1) \zeta]^{h}\right. \\
& \left.(p+w) l_{p+w} j_{p+w} z^{p+w}\right]\left(1+\varrho e^{i \theta}\right)+\left[p^{h+1}(p-1) z^{p}-\sum_{w=1}^{\infty}[1+(p+w+\phi-\gamma-1) \zeta]^{h}\right. \\
& \left.(p+w)(p+w-1) l_{p+w} j_{p+w} z^{p+w}\right] \beta\left(1+\varrho e^{i \theta}\right)-\left[p^{n} z^{p}-\sum_{w=1}^{\infty}[1+(p+w+\phi-\gamma-1) \zeta]^{h}\right. \\
& \left.l_{p+w} j_{p+w} z^{p+w}\right] \varrho e^{i \theta}+(1-\psi)\left[p^{n} z^{p}-\sum_{w=1}^{\infty}[1+(p+w+\phi-\gamma-1) \zeta]^{h} l_{p+w} j_{p+w} z^{p+w}\right] \mid \\
& =\mid p^{h+1} z^{p}+p^{h+1} z^{p} \varrho e^{i \theta}-\sum_{w=1}^{\infty}[1+(p+w+\phi-\gamma-1) \zeta]^{h}(p+w)\left(1+\varrho e^{i \theta}\right) l_{p+w} j_{p+w} z^{p+w} \\
& +p^{h+1}(p-1) z^{p} \beta\left(1+\varrho e^{i \theta}\right)-\sum_{w=1}^{\infty}[1+(p+w+\phi-\gamma-1) \zeta]^{h}(p+w)(p+w-1)\left(1+\varrho e^{i \theta}\right) \\
& \beta l_{p+w} j_{p+w} z^{p+w}-p^{h} z^{p} \varrho e^{i \theta}+\sum_{w=1}^{\infty}[1+(p+w+\phi-\gamma-1) \zeta]^{h} \varrho e^{i \theta} l_{p+w} j_{p+w} z^{p+w}+p^{h} z^{p}-\psi p^{h} z^{p} \\
& -(1-\psi) \sum_{w=1}^{\infty}[1+(p+w+\phi-\gamma-1) \zeta]^{h} l_{p+w} j_{p+w} z^{p+w} \mid \\
& =\mid p^{h} z^{p}[p-\psi+1]+p^{h} z^{p} \varrho e^{i \theta}[p-1]+p^{h+1}(p-1) z^{p} \beta\left(1+\varrho e^{i \theta}\right)-\sum_{w=1}^{\infty}\left[(p+w)\left(1+\varrho e^{i \theta}\right)\right. \\
& \left.+\beta(p+w)(p+w-1)\left(1+\varrho e^{i \theta}\right)-\varrho e^{i \theta}+(1-\psi)\right]\left[[1+(p+w+\phi-\gamma-1) \zeta]^{h} l_{p+w} j_{p+w} z^{p+w}\right] \mid .
\end{aligned}
$$


Now with $|z|=1$

$$
\begin{array}{r}
\geq p^{h}[p-\psi+1]+p^{h} \varrho[p-1]+p^{h+1}(p-1) \beta(1+\varrho)-\sum_{w=1}^{\infty}[(p+w)[1+\beta(p+w-1)](1+\varrho) \\
-\varrho+(1-\psi)]\left[[1+(p+w+\phi-\gamma-1) \zeta]^{h} l_{p+w} j_{p+w}\right] \cdot \quad(2.3)
\end{array}
$$

Also,

$$
\begin{aligned}
& |\mathcal{A}(z)-(1+\psi) \mathcal{B}(z)|=\mid\left[p^{h+1} z^{p}-\sum_{w=1}^{\infty}[1+(p+w+\phi-\gamma-1) \zeta]^{h}\right. \\
& \left.(p+w) l_{p+w} j_{p+w} z^{p+w}\right]\left(1+\varrho e^{i \theta}\right)+\left[p^{h+1}(p-1) z^{p}-\sum_{w=1}^{\infty}[1+(p+w+\phi-\gamma-1) \zeta]^{h}\right. \\
& \left.(p+w)(p+w-1) l_{p+w} j_{p+w} z^{p+w}\right] \beta\left(1+\varrho e^{i \theta}\right)-\left[p^{n} z^{p}-\sum_{w=1}^{\infty}[1+(p+w+\phi-\gamma-1) \zeta]^{h}\right. \\
& \left.l_{p+w} j_{p+w} z^{p+w}\right] \varrho e^{i \theta}-(1+\psi)\left[p^{n} z^{p}-\sum_{w=1}^{\infty}[1+(p+w+\phi-\gamma-1) \zeta]^{h} l_{p+w} j_{p+w} z^{p+w}\right] \mid \\
& =\mid p^{h+1} z^{p}+p^{h+1} z^{p} \varrho e^{i \theta}-\sum_{w=1}^{\infty}[1+(p+w+\phi-\gamma-1) \zeta]^{h}(p+w)\left(1+\varrho e^{i \theta}\right) l_{p+w} j_{p+w} z^{p+w} \\
& +p^{h+1}(p-1) z^{p} \beta\left(1+\varrho e^{i \theta}\right)-\sum_{w=1}^{\infty}[1+(p+w+\phi-\gamma-1) \zeta]^{h}(p+w)(p+w-1)\left(1+\varrho e^{i \theta}\right) \\
& \beta l_{p+w} j_{p+w} z^{p+w}-p^{h} z^{p} \varrho e^{i \theta}+\sum_{w=1}^{\infty}[1+(p+w+\phi-\gamma-1) \zeta]^{h} \varrho e^{i \theta} l_{p+w} j_{p+w} z^{p+w}-p^{h} z^{p}-\psi p^{h} z^{p} \\
& -(1-\psi) \sum_{w=1}^{\infty}[1+(p+w+\phi-\gamma-1) \zeta]^{h} l_{p+w} j_{p+w} z^{p+w} \mid \\
& =\mid p^{h} z^{p}[p-\psi-1]+p^{h} z^{p} \varrho e^{i \theta}[p-1]+p^{h+1}(p-1) z^{p} \beta\left(1+\varrho e^{i \theta}\right)-\sum_{w=1}^{\infty}\left[(p+w)\left(1+\varrho e^{i \theta}\right)\right. \\
& \left.+\beta(p+w)(p+w-1)\left(1+\varrho e^{i \theta}\right)-\varrho e^{i \theta}-(1+\psi)\right]\left[[1+(p+w+\phi-\gamma-1) \zeta]^{h} l_{p+w} j_{p+w} z^{p+w}\right] \mid .
\end{aligned}
$$


Now with $|z|=1$

$$
\begin{array}{r}
\leq p^{h}[\psi+1-p]+p^{h} \varrho[p-1]+p^{h+1}(p-1) \beta(1+\varrho)+\sum_{w=1}^{\infty}[(p+w)[1+\beta(p+w-1)](1+\varrho) \\
-\varrho-(1+\psi)]\left[[1+(p+w+\phi-\gamma-1) \zeta]^{h} l_{p+w} j_{p+w}\right] . \quad(2.4)
\end{array}
$$

It is easy to show that

$$
\begin{gathered}
|\mathcal{A}(z)+(1-\psi) \mathcal{B}(z)|-|\mathcal{A}(z)-(1+\psi) \mathcal{B}(z)|=2 p^{h}[p-\psi]-2 \sum_{w=1}^{\infty}[(p+w)[1+\beta(p+w-1)] \\
(1+\varrho)-(\varrho+\psi)]\left[[1+(p+w+\phi-\gamma-1) \zeta]^{h} l_{p+w} j_{p+w}\right] \geq 0 \\
-2 \sum_{w=1}^{\infty}[(p+w)[1+\beta(p+w-1)](1+\varrho)-(\varrho+\psi)]\left[[1+(p+w+\phi-\gamma-1) \zeta]^{h} l_{p+w} j_{p+w}\right] \\
\geq-2 p^{h}[p-\psi] \\
\sum_{w=1}^{\infty}[(p+w)[1+\beta(p+w-1)](1+\varrho)-(\varrho+\psi)]\left[[1+(p+w+\phi-\gamma-1) \zeta]^{h} l_{p+w} j_{p+w}\right] \\
\leq p^{h}[p-\psi] .
\end{gathered}
$$

Conversely, suppose the inequality (2.5) holds, we need to show that

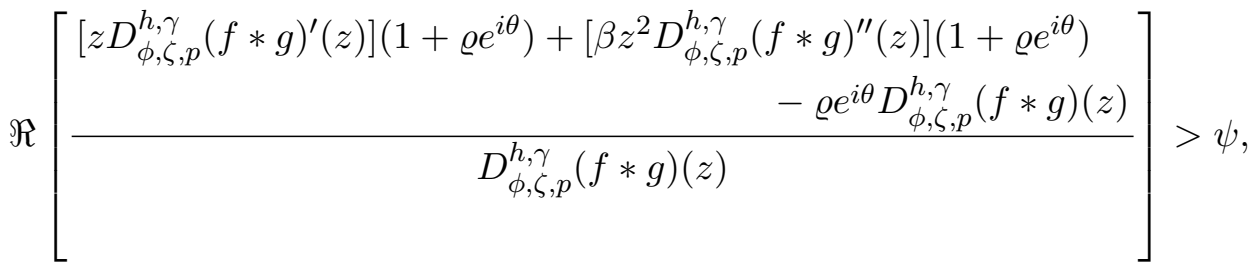

$$
\begin{aligned}
& \Re\left[\begin{array}{c}
{\left[z D_{\phi, \zeta, p}^{h, \gamma}(f * g)^{\prime}(z)\right]\left(1+\varrho e^{i \theta}\right)+\left[\beta z^{2} D_{\phi, \zeta, p}^{h, \gamma}(f * g)^{\prime \prime}(z)\right]\left(1+\varrho e^{i \theta}\right)} \\
-\varrho e^{i \theta} D_{\phi, \zeta, p}^{h, \gamma}(f * g)(z)-\psi D_{\phi, \zeta, p}^{h, \gamma}(f * g)(z) \\
D_{\phi, \zeta, p}^{h, \gamma}(f * g)(z)
\end{array}\right]>\psi .
\end{aligned}
$$


Since $\left|e^{i \theta}\right|=1$, hence $\Re\left(e^{i \theta}\right) \leq\left|e^{i \theta}\right|=1$, letting $|z| \longrightarrow 1^{-1}$ yields, we let $H=$ $[1+(p+w+\phi-\gamma-1) \zeta]^{h}$

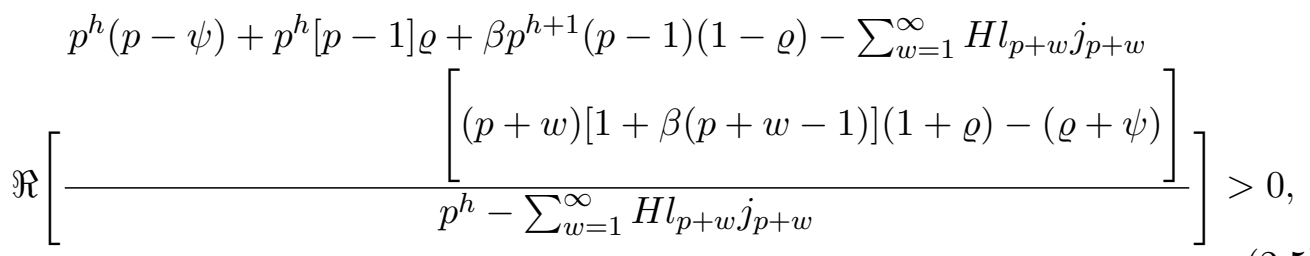

then we have

$$
\begin{aligned}
& \sum_{w=1}^{\infty} H l_{p+w} j_{p+w}[(p+w)[1+\beta(p+w-1)](1+\varrho)-(\varrho+\psi)] \leq p^{h}(p-\psi)+p^{h}[p-1] \varrho \\
& +\beta p^{h+1}(p-1) z^{p}(1-\varrho) \\
& f(z)=z-\frac{p^{h}(p-\psi)+p^{h}[p-1] \varrho+\beta p^{h+1}(p-1) z^{p}(1-\varrho)}{\sum_{w=1}^{\infty}[1+(p+w+\phi-\gamma-1) \zeta]^{h}} \\
& {[(p+w)[1+\beta(p+w-1)](1+\varrho)-(\varrho+\psi)] j_{p+w}}
\end{aligned}
$$

which completes the proof.

Corollary 2.4. Let $f(z) \in \mathcal{R}^{*}(p, g, \psi, \varrho, \beta, \phi, \gamma, \zeta)$. Then

$$
l_{p+w} \leq \frac{p^{h}(p-\psi)+p^{h}[p-1] \varrho+\beta p^{h+1}(p-1) z^{p}(1-\varrho)}{[1+(p+w+\phi-\gamma-1) \zeta]^{h}[(p+w)[1+\beta(p+w-1)](1+\varrho)-(\varrho+\psi)] j_{p+w}} .
$$

Taking $\beta=0$ in Theorem 2.3 , we have the following corollary.

Corollary 2.5. Let $f(z) \in \mathcal{T}_{p}$. Then $f(z) \in \mathcal{R}^{*}(p, g, \psi, \varrho, \beta, \phi, \gamma, \zeta)$ if and only if

$$
\sum_{w=1}^{\infty}\{(p+k)(1+\psi)-(\varrho+\psi)\}[1+(p+w+\phi-\gamma-1) \zeta]^{h} \leq p^{h}[p-\psi] .
$$




\section{Growth Theorem and Distortion Theorem}

Theorem 3.1. If $f(z) \in \mathcal{R}^{*}(p, g, \psi, \varrho, \beta, \phi, \gamma, \zeta)$ and $j_{p+w} \geq j_{2}$, then

$$
\begin{aligned}
& r^{p}-\frac{p^{h}(p-\psi)}{[(p+1)[1+\beta p](1+\varrho)-(\varrho+\psi)][1+(p+\phi-\gamma) \zeta]^{h} j_{p+1}} r^{p+1} \leq|f(z)| \\
& \quad \leq r^{p}+\frac{p^{h}(p-\psi)}{[(p+1)[1+\beta p](1+\varrho)-(\varrho+\psi)][1+(p+\phi-\gamma) \zeta]^{h} j_{p+1}} r^{p+1}
\end{aligned}
$$

and

$$
\begin{aligned}
& p r^{p-1}-\frac{(p+1) p^{h}(p-\psi)}{[(p+1)[1+\beta p](1+\varrho)-(\varrho+\psi)][1+(p+\phi-\gamma) \zeta]^{h} j_{p+1}} r^{p} \leq|f(z)| \\
& \leq p r^{p-1}+\frac{(p+1) p^{h}(p-\psi)}{[(p+1)[1+\beta p](1+\varrho)-(\varrho+\psi)][1+(p+\phi-\gamma) \zeta]^{h} j_{p+1}} r^{p} .
\end{aligned}
$$

The result is sharp for, $(|z|=r<1)$

$$
f(z)=z^{p}-\frac{p^{h}(p-\psi)}{[(p+1)[1+\beta p](1+\varrho)-(\varrho+\psi)][1+(p+\phi-\gamma) \zeta]^{h} j_{p+1}} r^{p+1} .
$$

Proof. Since

$$
f(z)=z^{p}-\sum_{w=1}^{\infty} l_{p+w} z^{p+w},
$$

we have

$$
\begin{aligned}
& |f(z)|=\left|z^{p}-\sum_{w=1}^{\infty} l_{p+w} z^{p+w}\right| \leq|z|^{p}+\sum_{w=1}^{\infty} l_{p+w}|z|^{p+w} \leq r^{p}+r^{p+1} \sum_{w=1}^{\infty} l_{p+w} \\
& {[(p+1)[1+\beta p](1+\varrho)-(\varrho+\psi)][1+(p+\phi-\gamma) \zeta]^{h} j_{p+1}} \\
& \leq[(p+w)[1+\beta(p+w-1)](1+\varrho)-(\varrho+\psi)][1+(p+w+\phi-\gamma-1) \zeta]^{h} j_{p+w} .
\end{aligned}
$$

Using Theorem 2.3, we have

$$
\begin{aligned}
& {[(p+1)[1+\beta p](1+\varrho)-(\varrho+\psi)][1+(p+\phi-\gamma) \zeta]^{h} j_{p+1} \sum_{w=1}^{\infty} l_{p+w} } \\
& \leq \sum_{w=1}^{\infty} l_{p+w} j_{p+w}[(p+w)[1+\beta(p+w-1)](1+\varrho)-(\varrho+\psi)][1+(p+w+\phi-\gamma-1) \zeta]^{h} j_{p+w} \\
& \leq p^{h}(p-\psi)
\end{aligned}
$$


that is

$$
\sum_{w=1}^{\infty} l_{p+w} \leq \frac{p^{h}(p-\psi)}{[(p+1)[1+\beta p](1+\varrho)-(\varrho+\psi)][1+(p+\phi-\gamma) \zeta]^{h} j_{p+1}}
$$

using the above equation in 3.2 , we have

$$
|f(z)| \leq r^{p}+\frac{p^{h}(p-\psi)}{[(p+1)[1+\beta p](1+\varrho)-(\varrho+\psi)][1+(p+\phi-\gamma) \zeta]^{h} j_{p+1}} r^{p+1}
$$

and

$$
|f(z)| \geq r^{p}-\frac{p^{h}(p-\psi)}{[(p+1)[1+\beta p](1+\varrho)-(\varrho+\psi)][1+(p+\phi-\gamma) \zeta]^{h} j_{p+1}} r^{p+1} .
$$

The result is sharp for

$$
|f(z)|=z^{p}-\frac{p^{h}(p-\psi)}{[(p+1)[1+\beta p](1+\varrho)-(\varrho+\psi)][1+(p+\phi-\gamma) \zeta]^{h} j_{p+1}} r^{p+1} .
$$

Similarly, since

$$
f^{\prime}(z)=p z^{p-1}-\sum_{w=1}^{\infty}(p+w) l_{p+w} z^{p+w-1}
$$

we have that

$$
\begin{gathered}
\left|f^{\prime}(z)\right|=\left|p z^{p-1}-\sum_{w=1}^{\infty}(p+w) l_{p+w} z^{p+w-1}\right| \leq p|z|^{p-1}+\sum_{w=1}^{\infty}(p+w) l_{p+w}|z|^{p+w-1} \\
\leq p r^{p-1}+(p+1) r^{p} \sum_{w=1}^{\infty} l_{p+w} \quad(3.2) \\
{[(p+1)[1+\beta p](1+\varrho)-(\varrho+\psi)][1+(p+\phi-\gamma) \zeta]^{h} j_{p+1}} \\
\leq[(p+w)[1+\beta(p+w-1)](1+\varrho)-(\varrho+\psi)][1+(p+w+\phi-\gamma-1) \zeta]^{h} j_{p+w} .
\end{gathered}
$$

Using Theorem 2.3, we have

$$
\begin{aligned}
& {[(p+1)[1+\beta p](1+\varrho)-(\varrho+\psi)][1+(p+\phi-\gamma) \zeta]^{h} j_{p+1} \sum_{w=1}^{\infty} l_{p+w} } \\
& \leq \sum_{w=1}^{\infty} l_{p+w} j_{p+w}[(p+w)[1+\beta(p+w-1)](1+\varrho)-(\varrho+\psi)][1+(p+w+\phi-\gamma-1) \zeta]^{h} j_{p+w} \\
& \leq p^{h}(p-\psi)
\end{aligned}
$$


that is

$$
\sum_{w=1}^{\infty} l_{p+w} \leq \frac{p^{h}(p-\psi)}{[(p+1)[1+\beta p](1+\varrho)-(\varrho+\psi)][1+(p+\phi-\gamma) \zeta]^{h} j_{p+1}}
$$

using the above equation in 3.2 ,

$$
\left|f^{\prime}(z)\right| \leq p r^{p-1}+\frac{(p+1) p^{h}(p-\psi)}{[(p+1)[1+\beta p](1+\varrho)-(\varrho+\psi)][1+(p+\phi-\gamma) \zeta]^{h} j_{p+1}} r^{p}
$$

and

$$
\begin{gathered}
\left|f^{\prime}(z)\right| \geq p r^{p-1}-\frac{(p+1) p^{h}(p-\psi)}{[(p+1)[1+\beta p](1+\varrho)-(\varrho+\psi)][1+(p+\phi-\gamma) \zeta]^{h} j_{p+1}} r^{p} \\
\left|f^{\prime}(z)\right| \geq 1-\frac{(p+1) p^{h}(p-\psi)}{[(p+1)[1+\beta p](1+\varrho)-(\varrho+\psi)][1+(p+\phi-\gamma) \zeta]^{h} j_{p+1}} r^{p}
\end{gathered}
$$

This completes the proof.

\section{Radii of Univalent Starlikeness, Convexity and Close to Convexity}

Theorem 4.1. If $f(z) \in \mathcal{R}^{*}(p, g, \psi, \varrho, \beta, \phi, \gamma, \zeta)$, then $f(z)$ is starlike of order $\sigma(0 \leq \sigma<1)$ in the disc $|z|<r_{1}(p, g, \psi, \varrho, \beta, \phi, \gamma, \zeta, \sigma)$, where

$r_{1}(p, g, \psi, \varrho, \beta, \phi, \gamma, \zeta, \sigma)=$

$i n f_{p+w}\left\{\frac{(1-\sigma)[(p+w)[1+\beta(p+w-1)](1+\varrho)-(\varrho+\psi)]}{(p+w-\sigma)\left[p^{h}(p-\psi)+p^{h}[p-1] \varrho+\beta p^{h+1}(p-1) z^{p}(1-\varrho)\right]}\right\}^{\frac{1}{p+w-1}}$

where $p \in \mathbb{N}=\{1,2,3, \cdots\}$.

Proof. It is sufficient to show that

$$
\left|\frac{z f^{\prime}(z)}{f(z)}-1\right| \leq 1-\sigma \quad(0 \leq \sigma<1)
$$


for $|z|<r_{1}(p, g, \psi, \varrho, \beta, \phi, \gamma, \zeta, \sigma)$, we have

$$
\begin{aligned}
\left|\frac{z f^{\prime}(z)}{f(z)}-1\right| & =\left|\frac{z\left[p z^{p-1}-\sum_{w=1}^{\infty}(p+w) l_{p+w} z^{p+w-1}\right]}{z\left[z^{p-1}-\sum_{w=1}^{\infty} l_{p+w} z^{p+w-1}\right]}-1\right| \\
& =\left|\frac{p z^{p-1}-\sum_{w=1}^{\infty}(p+w) l_{p+w} z^{p+w-1}}{z^{p-1}-\sum_{w=1}^{\infty} l_{p+w} z^{p+w-1}}-1\right| \\
& =\left|\frac{\left[p z^{p-1}-\sum_{w=1}^{\infty}(p+w) l_{p+w} z^{p+w-1}\right]-\left[z^{p-1}-\sum_{w=1}^{\infty} l_{p+w} z^{p+w-1}\right]}{z^{p-1}-\sum_{w=1}^{\infty} l_{p+w} z^{p+w-1}}\right| \\
& =\left|\frac{p z^{p-1}-\sum_{w=1}^{\infty}(p+w) l_{p+w} z^{p+w-1}-z^{p-1}+\sum_{w=1}^{\infty} l_{p+w} z^{p+w-1}}{z^{p-1}-\sum_{w=1}^{\infty} l_{p+w} z^{p+w-1}}\right| \\
& =\frac{(p-1) z^{p-1}-\sum_{w=1}^{\infty}(p+w-1) l_{p+w} z^{p+w-1}}{z^{p-1}-\sum_{w=1}^{\infty} l_{p+w} z^{p+w-1}} \leq 1-\sigma
\end{aligned}
$$

if

$$
\sum_{w=1}^{\infty} \frac{(p+w-\sigma) l_{p+w}|z|^{p+w-1}}{1-\sigma} \leq 1 .
$$

Hence by Theorem 2.3, (4.2) will be true if

$$
\begin{aligned}
& \frac{(p+w-\sigma) l_{p+w}|z|^{p+w-1}}{1-\sigma} \leq \\
& \frac{[(p+w)[1+\beta(p+w-1)](1+\varrho)-(\varrho+\psi)][1+(p+w+\phi-\gamma-1) \zeta]^{h} j_{p+w}}{\left[p^{h}(p-\psi)+p^{h}[p-1] \varrho+\beta p^{h+1}(p-1) z^{p}(1-\varrho)\right]}
\end{aligned}
$$

and hence

$$
|z| \leq\left\{\frac{(1-\sigma)[(p+w)[1+\beta(p+w-1)](1+\varrho)-(\varrho+\psi)]}{(p+w-\sigma)\left[p^{h}(p-\psi)+p^{h}[p-1] \varrho+\beta p^{h+1}(p-1) z^{p}(1-\varrho)\right]}\right\}^{\frac{1}{p+w-1}}
$$

setting

$$
|z|=r_{1}(p, g, \psi, \varrho, \beta, \phi, \gamma, \zeta, \sigma)
$$

we get the desired result. 
Theorem 4.2. If $f(z) \in \mathcal{R}^{*}(p, g, \psi, \varrho, \beta, \phi, \gamma, \zeta)$, then $f(z)$ is convex of order $\sigma(0 \leq \sigma<1)$ in the disc $|z|<r_{2}(p, g, \psi, \varrho, \beta, \phi, \gamma, \zeta, \sigma)$, where

$r_{2}(p, g, \psi, \varrho, \beta, \phi, \gamma, \zeta, \sigma)=$

$i n f_{p+w}\left\{\frac{(1-\sigma)[(p+w)[1+\beta(p+w-1)](1+\varrho)-(\varrho+\psi)]}{(p+w)(p+w-\sigma)\left[p^{h}(p-\psi)+p^{h}[p-1] \varrho+\beta p^{h+1}(p-1) z^{p}(1-\varrho)\right]}\right\}^{\frac{1}{p+w-1}}$,

where $p \in \mathbb{N}=\{1,2,3, \cdots\}$.

Proof. It is sufficient to show that

$$
\begin{aligned}
\left|\frac{z f^{\prime \prime}(z)}{f^{\prime}(z)}+1-1\right| & =\left|\frac{p(p-1) z^{p-1}-\sum_{w=1}^{\infty}(p+w)(p+w-1) l_{p+w} z^{p+w-1}}{p z^{p-1}-\sum_{w=1}^{\infty}(p+w) l_{p+w} z^{p+w-1}}\right| \\
& =\frac{p(p-1)-\sum_{w=1}^{\infty}(p+w)(p+w-1) l_{p+w} z^{p}}{p-\sum_{w=1}^{\infty}(p+w) l_{p+w} z^{p}} \leq 1-\sigma
\end{aligned}
$$

then

$$
\sum_{w=1}^{\infty} \frac{(p+w)(p+w-\sigma) l_{p+w}|z|^{p+w-1}}{1-\sigma} \leq 1-\sigma .
$$

Hence, by Theorem 2.3, 4.5 will be

$$
\begin{gathered}
\frac{(p+w)(p+w-\sigma) l_{p+w}|z|^{p+w-1}}{1-\sigma} \leq \frac{1}{\sum_{w=1}^{\infty} l_{p+w}} \\
\frac{(p+w)(p+w-\sigma) l_{p+w}|z|^{p+w-1}}{1-\sigma} \leq \\
\frac{[(p+w)[1+\beta(p+w-1)](1+\varrho)-(\varrho+\psi)][1+(p+w+\phi-\gamma-1) \zeta]^{h} j_{p+w}}{\left[p^{h}(p-\psi)+p^{h}[p-1] \varrho+\beta p^{h+1}(p-1) z^{p}(1-\varrho)\right]}
\end{gathered}
$$

and hence

$$
|z| \leq\left\{\begin{array}{c}
(1-\sigma)[(p+w)[1+\beta(p+w-1)](1+\varrho)-(\varrho+\psi)] \\
{\left[p^{h}(p-\psi)+p^{h}[p-1] \varrho+\beta p^{h+1}(p-1) z^{p}(1-\varrho)\right]}
\end{array}\right\}
$$


setting

$$
|z|=r_{2}(p, g, \psi, \varrho, \beta, \phi, \gamma, \zeta, \sigma)
$$

we get the desired result.

Theorem 4.3. If $f(z) \in \mathcal{R}^{*}(p, g, \psi, \varrho, \beta, \phi, \gamma, \zeta)$, then $f(z)$ is close to convex of order $\sigma(0 \leq \sigma<1)$ in the disc $|z|<r_{3}(p, g, \psi, \varrho, \beta, \phi, \gamma, \zeta, \sigma)$, where

$$
\begin{aligned}
& r_{3}(p, g, \psi, \varrho, \beta, \phi, \gamma, \zeta, \sigma)= \\
& \quad \inf _{p+w}\left\{\begin{array}{c}
(1-\sigma)[(p+w)[1+\beta(p+w-1)](1+\varrho)-(\varrho+\psi)] \\
\frac{[1+(p+w+\phi-\gamma-1) \zeta]^{h} j_{p+w}}{(p+w)\left[p^{h}(p-\psi)+p^{h}[p-1] \varrho+\beta p^{h+1}(p-1) z^{p}(1-\varrho)\right]}
\end{array}\right\}^{\frac{1}{p+w-1}},
\end{aligned}
$$

where $p \in \mathbb{N}=\{1,2,3, \cdots\}$.

Proof. It is sufficient to show that

$$
\left|f^{\prime}(z)-1\right| \leq 1-\sigma \quad(0 \leq \sigma<1)
$$

for $|z| \leq r_{3}(p, g, \psi, \varrho, \beta, \phi, \gamma, \zeta, \sigma)$ we have

$$
\begin{aligned}
\left|f^{\prime}(z)-1\right|=\left|p z^{p-1}-\sum_{w=1}^{\infty}(p+w) l_{p+w} z^{p+w-1}-1\right| \leq p|z|^{p-1} \\
-\sum_{w=1}^{\infty}(p+w) l_{p+w}|z|^{p+w-1}-1 \leq 1-\sigma .
\end{aligned}
$$

If

$$
\sum_{w=1}^{\infty} \frac{(p+w) l_{p+w}|z|^{p+w-1}}{1-\sigma} \leq 1,
$$

then by Theorem 2.3. (4.8) will be true if

$$
\begin{aligned}
& \frac{(p+w) l_{p+w}|z|^{p+w-1}}{1-\sigma} \leq \\
& \frac{[(p+w)[1+\beta(p+w-1)](1+\varrho)-(\varrho+\psi)][1+(p+w+\phi-\gamma-1) \zeta]^{h} j_{p+w}}{\left[p^{h}(p-\psi)+p^{h}[p-1] \varrho+\beta p^{h+1}(p-1) z^{p}(1-\varrho)\right]}
\end{aligned}
$$


and hence

$$
|z| \leq\left\{\frac{[(p+w)[1+\beta(p+w-1)](1+\varrho)-(\varrho+\psi)]}{(p+w)\left[p^{h}(p-\psi)+p^{h}[p-1] \varrho+\beta p^{h+1}(p-1) z^{p}(1-\varrho)\right]}\right\}^{\frac{1}{p+w-1}}
$$

setting

$$
|z|=r_{3}(p, g, \psi, \varrho, \beta, \phi, \gamma, \zeta, \sigma)
$$

we get the desired result. The proof is complete.

Remark 4.4. If we put $p=1$ in Theorems 2.3, 3.1 and 4.1, we obtain the corresponding result studied by Godwin and Opoola [7].

\section{References}

[1] W. G. Atshan and R. H. Buti, Fractional calculus of class of univalent functions with negative coefficient defined by Hadamard product with Rafid operator, European Pure Appl. Math. 4(2) (2010), 162-173.

[2] W. G. Atshan, A. J. M. Khalaf and M. M Mahdi, On a new subclass of univalent functions with negative coefficient defined by Hadamard product, European Journal of Scientific Research 119(3) (2014), 462-472.

[3] H. E. Darwish, A. Y. Lashin and E. M. Madar, On certain classes of univalent functions with negative coefficients by defined convulation, Electronic Journal of Mathematical Analysis and Applications 4(1) (2016), 143-154.

[4] J. Dziok and H. M. Srivastava, Certain subclasses of analytic functions associated with the generalized hypergeomtric function, Integral Transforms Spec. Funct. 14(1) (2003), 7-18. https://doi.org/10.1080/10652460304543

[5] A. W. Goodman, Univalent functions and non-analytic curves, Proc. Amer. Math. Soc. 8 (1957), 598-601. https://doi.org/10.1090/S0002-9939-1957-0086879-9

[6] T. O. Opoola, On a subclass of univalent function defined by generalized differential operator, Internat. J. Math. Anal. 11(18) (2017), 869-876. https://doi.org/10.12988/ijma.2017.7232 
[7] E. C. Godwin and T. O. Opoola, On a new subclass of univalent function with negative coefficients using a generalized different operator, Internat. J. Math. Anal. 11(18) (2017), 869-876. https://doi.org/10.12988/ijma.2017.7232

[8] S. Ruscheweyh, New criteria for univalent functions, Proc. Amer. Math. Soc. 49 (1975), 109-115. https://doi.org/10.1090/S0002-9939-1975-0367176-1

[9] H. Silverman, Univalent functions with negative coefficient, Proc. Amer. Math. Soc. 51 (1975), 109-116. https://doi.org/10.1090/S0002-9939-1975-0369678-0

[10] H. Silverman, Integral means for univalent function with negative coefficients, Houston J. Math. 23(1) (1997), 169-174.

[11] T. G. Shaba, A. A. Ibrahim and M. F. Oyedotun, A new subclass of analytic functions defined by Opoola differential operator, Advances in Mathematics Scientific Journal 9(7) (2020), 4829-4841. https://doi.org/10.37418/amsj.9.8.5

[12] T. G. Shaba, On some new subclass of bi-univalent functions associated with Opoola differential operator, Open J. Math. Anal. 4(2) (2020), 74-79. https://doi.org/10.30538/psrp-oma2020.0064

This is an open access article distributed under the terms of the Creative Commons Attribution License (http://creativecommons.org/licenses/by/4.0/), which permits unrestricted, use, distribution and reproduction in any medium, or format for any purpose, even commercially provided the work is properly cited. 